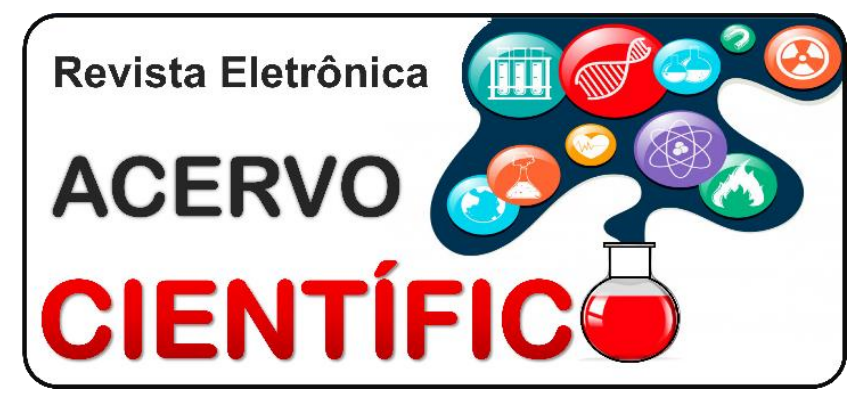

REVISÃO BIBLIOGRÁFICA

\title{
Complicações da revascularização do miocárdio em pacientes com diabetes mellitus
}

Complications of myocardial revascularization in patients with diabetes mellitus

Complicaciones de la revascularización miocárdica en pacientes con diabetes mellitus

Thalia do Nascimento Mota ${ }^{1 *}$, Gabriel Porto Soares ${ }^{2}$, Anamaria de Araújo Gomes ${ }^{3}$, Gabriela Gomes Vieira $^{4}$, Leo Nogueira Campos ${ }^{3}$, Mariana de Araújo Gomes ${ }^{5}$, Natany Ezequiel Santos ${ }^{4}$, Pauline Christina Campos Martins Ferreira ${ }^{3}$, Thayná Valentim Lopes ${ }^{4}$.

Resumo: Este artigo buscou elucidar a relação entre a ocorrência de complicações peri e pós operatórias da cirurgia de revascularização do miocárdio em pacientes portadores de diabetes mellitus (DM). A DM é uma doença de importância mundial, capaz de causar importantes danos ao sistema cardiovascular, aumentando significativamente o risco e a gravidade da doença aterosclerótica. Trata-se de uma revisão de literatura que avalia o prognóstico de pacientes portadores dessa doença crônica submetidos a diferentes métodos cirúrgicos de revascularização miocárdica, associado a outros fatores de risco. Os resultados apontam que pacientes com DM possuem maior risco de pior evolução após a cirurgia, entretanto, esse é um grupo heterogêneo de pacientes, e outras condições como esquema de tratamento hipoglicemiante, etnia, sexo, idade e história clínica, também contribuem para o desfecho. Conclui-se que a abordagem da doença arterial coronariana em portadores de DM depende de uma abordagem individualizada do paciente e de suas comorbidades.

Palavras-chave: Complicações do diabetes, Diabetes mellitus, Revascularização miocárdica.

\begin{abstract}
This article aimed to elucidate the relationship between the occurrence of perioperative and postoperative complications of surgical myocardial revascularization in patients with diabetes mellitus (DM). NDM is a disease of worldwide significance, capable of causing major damage to the cardiovascular system, increasing the risk and the severity of atherosclerotic disease. This is a review of the literature on the prognosis of patients with this chronic disease subjected to different surgical methods of myocardial revascularization, associated with other risk factors. The results have shown that patients with DM have a higher risk of worse evolution after surgery, however, this is a heterogeneous group of patients, and other conditions such as hypoglycemic treatment scheme, ethnicity, sex, age and clinical history, also contribute to the outcome. In conclusion, the approach to coronary artery disease in patients with DM depends on an individualized approach to the patient and his comorbidities.
\end{abstract}

Keywords: Diabetes complications, Diabetes mellitus, Myocardial revascularization.

\footnotetext{
1Universidad Autónoma San Sebastian (UASS), San Lorenzo - PY. *E-mail: thaliamarques225@gmail.com 2Universidade de Vassouras (UV), Vassouras - RJ.

${ }^{3}$ Universidade Federal de Ouro Preto (UFOP), Ouro Preto - MG.

${ }^{4}$ Universidade do Estado do Rio de Janeiro (UERJ), Rio de Janeiro - RJ.

${ }^{5}$ Centro Universitário de Belo Horizonte (UniBH), Belo Horizonte - MG.
} 
Resumen: Este artículo buscó dilucidar la relación entre la ocurrencia de complicaciones perioperatorias y postoperatorias de la cirugía de revascularización miocárdica en pacientes con diabetes mellitus (DM). La DM es una enfermedad de importancia mundial, capaz de causar un daño importante al sistema cardiovascular, aumentando significativamente el riesgo y la gravedad de la enfermedad aterosclerótica. Se trata de una revisión de la literatura que valora el pronóstico de los pacientes con esta enfermedad crónica sometidos a diferentes métodos quirúrgicos de revascularización miocárdica, asociados a otros factores de riesgo. Los resultados indican que los pacientes con DM tienen un mayor riesgo de peor evolución después de la cirugía, sin embargo, este es un grupo heterogéneo de pacientes, y otras condiciones como esquema de tratamiento hipoglucémico, etnia, sexo, edad e historia clínica, también contribuyen al resultado. Se concluye que el abordaje de la enfermedad arterial coronaria en pacientes con DM depende de un abordaje individualizado del paciente y sus comorbilidades.

Palabras clave: Complicaciones de la diabetes, Diabetes mellitus, Revascularización miocárdica.

\section{INTRODUÇÃO}

Diabetes mellitus (DM) é uma doença com relevância significativa global. Apresenta prevalência de 8,8\% na população mundial de 20 a 79 anos (intervalo de confiança de 95\%: 7,2 a 11,3), e tem potencial de crescimento de 203,1 milhões de afetados, em apenas 28 anos (IDF, 2017).

O diagnóstico da DM é baseado nos valores de glicose plasmática: valor de glicose em jejum maior ou igual a $126 \mathrm{mg} / \mathrm{dL}$; valor de glicemia após 2 horas de ingestão de 75 gramas de dextrosol maior ou igual a $200 \mathrm{mg} / \mathrm{dL}$, avaliado no teste de tolerância à glicose (TOTG); e hemoglobina glicada maior que 6,5\%. Como critério diagnóstico, os testes precisam ser realizados em duas ocasiões diferentes ou dois testes alterados na mesma amostra de sangue. Importante lembrar que valores de glicemia ao acaso maiores ou iguais a 200 $\mathrm{mg} / \mathrm{dL}$, associados a sintomas clássicos de hiperglicemia, já confirmam o diagnóstico de diabetes mellitus (ADA, 2019).

O tratamento pode ocorrer de três formas: farmacológica, cirúrgica e alterações do estilo de vida, como reeducação alimentar e prática de atividades físicas, sendo que estas podem estar associadas (MONTEIRO MA, et al., 2016). Os portadores de DM apresentam diversas anormalidades vasculares e metabólicas, como espessamento das membranas basais na microcirculação; disfunção endotelial com redução da síntese de vasodilatadores; elevação na produção de antiagregantes e alteração na matriz extracelular, o que leva a uma mudança na membrana basal dos vasos; maior adesividade das plaquetas em virtude do aumento de tromboxano A2 e redução de prostaciclina; e hiperviscosidade do sangue. Esse quadro é responsável por desencadear lesões em vasos e órgãos e, com isso, potencializar a susceptibilidade a infecções (FILHO B e BOGLIOLO G, 2016).

Além dessas anomalias, é visível que o miocárdio pode ser afetado diretamente pela DM, ocasionando a cardiomiopatia diabética (CD), sendo esta, se indevidamente tratada, possivelmente responsável por um comprometimento progressivo da função cardíaca. $\mathrm{Na} C D$, ocorrem anormalidades patológicas relacionadas às mitocôndrias. Estas têm a autofagia comprometida e, consequentemente, são acometidas por disfunções e por um processo de renovação defeituoso. Isso compromete a capacidade de redução-oxidação e energética dessas organelas e o acúmulo destas leva a um estresse oxidativo no miocárdio. Somado a isso, também há um comprometimento da alteração da ciclagem do cálcio, que com a evolução da doença leva a uma disfunção cardíaca diastólica e posteriormente a uma sistólica (AON MA e FOSTER BD, 2015). Somado a isso, é importante destacar que óbitos de pacientes com diabetes têm como motivação, principalmente, complicações cardiovasculares (BERRY C, et al., 2007).

Sabe-se que, em comparação com indivíduos sem DM, os pacientes diabéticos exibem quadros de maior extensão de doença coronariana ateromatosa (LAYERLE B e VIGNOLO W, 2017). Para tal, há diversas maneiras de tratamento, entre elas estão o controle dos fatores de risco e a cirurgia de revascularização miocárdica, sendo essa última, de acordo com as Diretrizes de Cirurgia de Revascularização Miocárdica, Valvopatias e Doenças da Aorta, o método mais comum para se tratar uma doença coronariana mais 
complexa. É indispensável considerar que, ainda que a diabetes, principalmente se mal controlada, influencie na resposta ao trauma de qualquer procedimento cirúrgico, ela não é uma contraindicação para a cirurgia de revascularização miocárdica, uma vez que os benefícios sobressaem (DIRETRIZES DE CIRURGIA DE REVASCULARIZAÇÃO MIOCÁRDICA, VALVOPATIAS E DOENÇAS DA AORTA, 2004).

O objetivo do presente estudo consiste em revisar a literatura científica sobre a cirurgia de revascularização do miocárdio em pacientes portadores de DM, buscando elucidar a relação entre a ocorrência de complicações peri e pós operatórias nos pacientes portadores dessa comorbidade crônica.

\section{REVISÃO BIBLIOGRÁFICA}

\section{Complicações gerais}

Em pacientes com DM, a doença arterial coronariana (DAC) tende a ser uma doença mais complexa, caracterizada por envolvimento multiarterial pequeno, difuso, calcificado e muitas vezes requer revascularização coronária. Embora a maioria dos ensaios clínicos comparando os resultados entre pacientes diabéticos com doença multiarterial tenham mostrado que a cirurgia de revascularização do miocárdio (CRM) foi superior à intervenção coronária percutânea (ICP) em termos de revascularização de repetição (RR), incidência de infarto do miocárdio (IM) e mortalidade, não é viável a realização de CRM em todos os pacientes diabéticos com doença multiarterial (WANG TKM, et al., 2017; NAITO R e KASAI T, 2015).

A partir disso, diversos estudos são feitos para analisar os resultados de pacientes diabéticos submetidos a CRM. O derrame, a insuficiência renal, a ventilação prolongada por mais de 24 horas, a infecção profunda da ferida esternal e a reoperação são as principais morbidade pós-operatórias que acometem os pacientes com DM submetidos a CRM. Além disso, a mortalidade operatória também ocorre em alguns casos, sendo associado ao coeficiente de variação da glicose no dia da cirurgia $(\mathrm{HbA} 1 \mathrm{c})$ e tratamento com insulina. No modelo de risco derivado da base de dados do Euro Score II, diabéticos em uso de insulina tiveram uma razão de chances maior de risco de mortalidade operatória do que aqueles que não usaram insulina (WANG TKM, et al., 2017).

Wang TKM, et al. (2017) relata que tratamentos para diabetes, incluindo estilo de vida, medicamentos orais e insulina, que reduzem a HbA1c, podem melhorar os resultados da CRM. No entanto, indivíduos diabéticos tipo $1 \mathrm{com} \mathrm{HbA1c}$ muito baixa possuem o dobro da mortalidade de controles pareados, o que significa que a redução excessiva de $\mathrm{HbA} 1 \mathrm{c}$ pode ser prejudicial. Embora não se tenha encontrado variáveis diabéticas para prever complicações pós-operatórias, alguns estudos relataram algumas associações, como a elevação de $\mathrm{HbA1c}$ estar relacionada a insuficiência renal aguda após CRM e outros estudos descobriram que a $\mathrm{HbA} 1 \mathrm{c}$ prediz a infecção da ferida esternal, que pode ser agravada pelo uso de enxertos de artéria mamária interna bilaterais.

\section{Mulheres e Reestenose}

Segundo Choles FE, et al. (2015), mulheres diabéticas têm mais lesões coronárias em comparação com mulheres não diabéticas. Há evidências de que as mulheres com DM apresentam resultados piores que os homens. Além disso, mulheres diabéticas com DAC submetidas a revascularização apresentam maior risco de reestenos devido às alterações fisiopatológicas em nível vascular, diminuição do efeito protetor dos estrógenos após a menopausa e ao fato do calibre de suas artérias ser menor que a dos não diabéticos e menor que a dos homens. Nelas a revascularização da artéria descendente anterior com cirurgia de revascularização está associada a maior incidência de resultados adversos em curto e médio prazo. Os resultados a longo prazo de morte ou IM são semelhantes para implante de stent e cirurgia. Porém, aos 5 anos, pacientes com stents necessitaram de maiores reoperações devido ao aparecimento de reestenose, o que indica a cirurgia de revascularização como técnica de escolha.

\section{Análise contemporânea das complicações}

Brush JE, et al. (2019), afirma que uma análise contemporânea das taxas de complicações de CRM em pacientes com DM é necessária porque a prevalência, a demografia e o tratamento dos pacientes com DM 
estão mudando. Nesse estudo, pacientes sem DM foram comparados com pacientes com DM, subdivididos de acordo com: se o DM foi ou não tratado com insulina antes da admissão. Foi mostrado que o status de diabetes no Grupo Sem Insulina foi um preditor significativo para AVC permanente, definido como um déficit neurológico confirmado que não foi resolvido em 24 horas e incluiu AVC isquêmico, AVC hemorrágico e AVC embólico. Já o status de diabetes no Grupo Insulina foi um preditor significativo para infecções do sítio cirúrgico e nova insuficiência renal, em que as infecções de sítio cirúrgico incluíram infecção de ferida esternal profunda e infecção de ferida de esternotomia superficial, e infecções de sítio cirúrgico pós-operatório, que incluíram infecções de esternotomia superficial e profunda, infecções local de coleta de conduto e infecções de canulação e a insuficiência renal foi definida em alguns casos como um aumento agudo na creatinina 3 vezes maior do que o valor basal ou $\geq 4 \mathrm{mg} / \mathrm{dl} \mathrm{e}$, em outros, como uma nova necessidade de diálise no pósoperatório. Porém, o status de DM não foi um preditor significativo de outras complicações pós-operatórias, isso porque tiveram outros preditores significativos como estado cirúrgico, idade, CRM prévia, doença arterial periférica, sexo, história de IAM prévio, fração de ejeção, raça e história de hipertensão.

\section{Ponte Aorta Coronária (PAC) X Angioplastia coronária (AC)}

O método utilizado para realizar a revascularização coronária pode apresentar diversos desfechos nos mais diferentes tipos de pacientes. Indivíduos diabéticos submetidos a (PAC) apresentaram menores taxas dos principais eventos cardíacos ou cerebrovasculares adversos (PECCA), IM não fatal e morte quando comparados ao grupo submetido à AC, cerca de $80 \%$ foram realizadas com Stents Eluidores de Drogas (SED), sendo a morte um fator que não apresentou significância estatística. Por outro lado, as taxas de Acidente Vascular Cerebral (AVC) se apresentaram significativamente maiores em pacientes submetido a PAC (RAMANATHAN K, et al., 2017).

Adicionalmente, outros estudos relativos à realização da PAC mostraram que pacientes com DM tratados com insulina apresentam maior risco de morte e outros eventos intra-hospitalares, além de ser preditor para infecção do sítio cirúrgico e surgimento de insuficiência renal quando comparados a pacientes sem DM; enquanto pacientes com DM controlada por outras formas que não a administração de insulina apresentam maior risco de eventos intra-hospitalares, mas não de morte, e também possuem maiores chances de ocorrência de um AVC permanente ao serem comparados ao grupo sem DM (BRUSH JE, et al., 2019).

Também foi estabelecido que, a curto prazo, a estratégia de revascularização tem impacto e varia de acordo com a acuidade de apresentação da doença de base, sendo a Síndrome Coronariana Aguda (SCA) ou DAC estável. O estudo determinou que entre os pacientes portadores de DM com SCA, a PAC foi fortemente favorecida em comparação com a AC em relação aos PECCA. Em contrapartida, pacientes portadores de DM com DAC estável não apresentaram diferenças significativas entre os dois procedimentos (RAMANATHAN K, et al., 2017).

O mesmo estudo também descreveu os desfechos a longo prazo, em que a relação entre a acuidade da doença de base (SCA ou DAC estável) e a escolha de tratamento não foi significante. Diante disso, pacientes com DM submetidos a PAC apresentaram desfechos mais favoráveis em relação ao risco de ocorrência dos PECCA e sua composição com a e revascularização repetida; morte por todas as causas; IM; AVC; e RR quando comparado a técnica de AC (RAMANATHAN K, et al., 2017).

Butaine AJ, et al. (2016) concluiu que a revascularização por ambas as técnicas em pacientes com DM e SCA apresenta melhores desfechos quando comparada ao manejo médico isoladamente e apresenta indicações semelhantes em quadros sem DM. Em relação a pacientes que apresentam DAC estável, com ou sem $\mathrm{DM}$, a revascularização de rotina não se mostrou benéfica.

\section{Uso de Stents eluidores de drogas (SED)}

A correta estratificação de risco para pacientes que serão submetidos à revascularização do miocárdio tem impacto direto na escolha do tratamento (PAC ou AC). Por meio de análises, foi possível demonstrar que ao serem tratados com a nova geração de SED, os pacientes com DM, em comparação com os sem DM, apresentavam um maior risco de revascularização da lesão-alvo e do vaso alvo, mas não foram encontradas diferenças em relação aos principais eventos cardíacos adversos (PECA). No entanto, em uma análise 
multivariada, o status da DM não foi relacionado com maiores riscos de morte cardíaca ou IM (KOSKINAS KC, et al., 2016).

Resultados a médio prazo demonstraram que não houve diferença de taxa dos PECCA; de desfecho composto por todas as causas de morte, IM e AVC; dos componentes dos PECCA individuais de morte; e IM entre os indivíduos com e sem DM submetidos a tratamento com SED de segunda geração. Também não houve relato de AVC nos dois grupos de pacientes. Por outro lado, ser portador de DM foi considerado fator de risco para necessidade de revascularização repetida. Além disso, foi demonstrado que os SED de nova geração podem contribuir para uma rápida endotelização, diminuição da inflamação e redução das taxas de trombose do Stent (LOUFTI M, et al., 2016).

\section{Stents eluidores de everolimus versus cirurgia de revascularização do miocárdio para pacientes com diabetes mellitus}

Bangalone S (2015) analisou pacientes com DM que foram submetidos à CRM ou ICP com stent eluidor de everolimus (EES). A curto prazo, o EES foi associado a um menor risco de morte e AVC, porém, maior risco de IM. A longo prazo, EES foi associado a risco semelhante de morte e risco menor de AVC, mas um risco maior de IM, nos indivíduos com revascularização incompleta (RI), e de RR comparados com a CRM. Estudos recentes indicam redução na morbidade e na mortalidade com stents mais novos, como o EES, quando comparados com stents antigos.

Enfim, o estudo atual reitera o excesso de risco inicial de CRM com aumento significativo de morte e AVC em 30 dias quando comparado com ICP. No entanto, a ICP com EES foi associada a risco semelhante de morte, a longo prazo, com a CRM. Além disso, também foi associada a risco aumentado de IM e RR, sem qualquer diferença de mortalidade quando comparada com CRM. Em pacientes com RC, o risco aumentado de IM com ICP não estava mais presente e a magnitude do aumento na RR diminuiu. Então, atualmente, a decisão entre ICP e CRM em pacientes com DM deve ser baseada na capacidade de obter revascularização completa $(\mathrm{RC})$ com ICP. Se ela não for alcançável, os pacientes devem ser considerados para CRM (BANGALONE S, 2015).

\section{Stents eluidores de Sirolimus (SES) versus Stents eluidores paclitaxel (PES)}

O uso de SED em pacientes diabéticos está associado a um menor risco de revascularização da lesãoalvo (TLR), sem qualquer aumento de morte ou IM, isso quando comparado com os stents convencionais (BMS). No entanto, existem algumas incertezas a respeito da eficácia de vários SED. Apesar da incidência menor de reestenose associada a SED em comparação com BMS, a anatomia coronariana mais complexa, o estado pró-trombótico, inflamatório e fatores de risco cardiovascular associados aos diabéticos são preditores independentes de desfechos clínicos desfavoráveis (BARTORELLI AL, et al., 2016; NAKAMURA M, et al., 2016).

Alguns estudos randomizados controlados revelaram a superioridade dos SES em comparação com os PES em termos de reestenose em pacientes com DM. Nesse estudo, foi feita uma análise em pacientes diabéticos submetidos a implante de stent coronário com SES ou PES, onde foram observadas uma taxa um pouco maior de PECCA, mortalidade, IM e AVC no grupo com SES quando comparado com o grupo PES. No que diz respeito a falha do vaso alvo (TVF), a taxa foi igual para ambos os grupos. No entanto, em 3 anos de análise, foi observado que a segurança e a eficácia dos SES e PES em pacientes com DM não deferiu (NAKAMURA M, et al., 2016).

\section{Comparação de desfechos com circulação extracorpórea versus sem circulação extracorpórea}

Um estudo mostrou que a CRM melhora a sobrevida de pacientes diabéticos com doença cardiovascular. A CRM tem sido realizada utilizando circulação extracorpórea (CEC) e sem CEC. No estudo foram analisados a mortalidade, IM e AVC (SINGH A, 2016).

As taxas de complicações intra-hospitalares foram menores entre pacientes submetidos à procedimentos sem CEC quando comparados aos com CEC. Porém, o desfecho composto de 30 dias de mortalidade, IM e AVC foi significativamente maior entre os pacientes sem CEC. A longo prazo, o risco de morte dos pacientes 
sem CEC foi um pouco maior. E, também, uma proporção significativamente maior de pacientes submetidos a revascularização sem CEC exigiram uma nova revascularização (SINGH A, 2016). Assim, o estudo sugere que a CRM sem CEC deve ser usada com cautela em pacientes com diabetes. Existe estudo recente, de apenas uma instituição, que, com um acompanhamento de 6 anos, mostra vantagem de sobrevida, a longo prazo, em pacientes que fizeram cirurgia com CEC (SINGH A, 2016).

A escolha do uso da bomba, circulação extracorpórea, ou sem bomba, fica a critério do cirurgião. Estudos foram feitos para avaliar o impacto fora da bomba versus com a bomba em pacientes com diabetes. Eles mostram que a taxa de permeabilidade do enxerto em um ano foi menor fora da bomba. Estes com CEC também eram mais propensos a RI. Há possibilidade de que a sobrevivência a longo prazo e os PECA podem ser piores fora da bomba. Os estudos também indicam que o uso do CEC favoreceu uma melhora na sobrevida após 5 anos da revascularização do miocárdio para pacientes diabéticos (SHROYER ALW, 2018).

Shroyer ALW (2014), relatou que para pacientes diabéticos tratados ativamente, isto é, que estavam recebendo hipoglicemiantes orais ou insulina ou ambos, CRM sem CEC não rendeu nenhuma vantagem sobre CRM com CEC.

Cirurgia sem CEC em pacientes diabéticos tratados ativamente resultou em menos RC. A curto prazo, a taxa de mortalidade operatória em 30 dias também mostrou uma tendência pior para fora da bomba. Em todo o coração houve uma diferença na eficácia geral da revascularização entre os procedimentos. Maior número de pacientes tratados ativamente que fizeram a revascularização com CEC foram efetivamente vascularizados em comparação com os que fizeram sem CEC (SHROYER ALW, 2014).

Enfim, há maiores vantagens com CEC para o enxerto. As taxas para a potência do enxerto para pacientes diabéticos tratados ativamente foram documentadas como sendo significativamente mais baixas no acompanhamento de um ano para o tratamento fora da bomba. Além disso, também foi identificado uma tendência de aumento da taxa de eventos adversos nestes pacientes tratados sem CEC (SHROYER ALW, 2014).

\section{CONSIDERAÇÕES FINAIS}

A partir da análise bibliográfica deste estudo, conclui-se que os pacientes com Diabetes Mellitus possuem maiores riscos de morbidade e mortalidade durante e após a CRM devido ao estado inflamatório e prótrombótico decorrente da doença. Ademais, as evidências demonstraram que os desfechos são variados e dependentes da DM estar descompensada ou tratada, seja com insulina ou outras medicações. Associado a isso, fatores como sexo, etnia, idade e história clínica de outras doenças também influenciam no prognóstico da doença arterial coronariana. Portanto, no que tange à escolha do método de revascularização, seja PAC ou AC, depende de uma abordagem individualizada das evidências disponíveis e do quadro clínico da doença de base e suas comorbidades, a fim de minimizar o risco de complicações e de mortalidade decorrentes do procedimento.

\section{REFERÊNCIAS}

1. AMERICAN DIABETES ASSOCIATION (ADA). 2.Classification and diagnosis of diabetes: Standarts of Medical Care in Diabetes - 2019. Diabetes Care, 2019, 42(1):S13-S28

2. AON MA, FOSTER BD. Diabetic cardiomyopathy and the role of mitochondrial dysfunction: Novel insights, mechanisms and therapeutic strategies. Antioxidants \& Redox Sinaling. 2015; 22: 1499-1501.

3. BANGALORE S, et al. Everolimus Eluting Stents Versus Coronary Artery Bypass Graft Surgery for Patients With Diabetes Mellitus and Multivessel Diseas. Circulation: Cardiovascular Interventions, 2015; 8(7): e002626

4. BARTORELLI AL, et al. One-Year Clinical Outcomes After Sirolimus-Eluting Coronary Stent Implantation in Diabetics Enrolled in the Worldwide e-SELECT Registry. Catheterization and Cardiovascular Interventions, 2016; 87(1): 52-62.

5. BERRY $\mathrm{C}$, et al. Coronary heart disease in patients with diabetes: part II: recent advances in coronary revascularization. J Am Coll Cardiol. 2007; 49:643-656

6. BRICK AV, et al. Diretrizes da cirúrgia de revascularização miocárdica valvopatias e doenças da aorta. Arq. Bras. Cardiol. [online]. 2004, 82: 1-20.

7. BRUSH JE, et al. Effect of Diabetes Mellitus on Complication Rates of Coronary Artery Bypass Grafting. The American journal of cardiology, 2019; 124(9): 1389-1396. 
8. BUNTAINE AJ, et al. Revascularization Strategies in Patients with Diabetes Mellitus and Acute Coronary Syndrome. Current cardiology reports, 2016; 18(8): 79.

9. CHOLES FE, et al. Reestenosis de la arteria coronaria descendenteanterior en mujeres diabéticas. Revista Colombiana de Cardiologia, 2016; 23(6): 545-551.

10. FILHO B, BOGLIOLO G. Patologia. 9 ed. Rio de Janeiro: Guanabara Koogan, 2016.

11. INTERNATIONAL DIABETES FEDERATION. IDF Diabetes Atlas. 8. ed. Bruxelas: International Diabetes Federation, 2017.

12. KOSKINAS KC, et al. Impact of diabetic status on outcomes after revascularization with drug-eluting stents in relation to coronary artery disease complexity: patient-level pooled analysis of 6081 patients. Circulation. Cardiovascular Interventions, 2016; 9(2): e003255.

13. LAYERLE B, VIGNOLO W. Tamizaje de cardiopatía isquémica y revascularización en pacientes diabéticos con enfermedad arterial coronaria estable: una perspectiva clínica. Rev. Urug. Cardiol., 2017, 32: 158 - 172.

14. LOUFTI M, et al. Outcomes of DES in diabetic and nondiabetic patients with complex coronary artery disease after risk stratification by the SYNTAX score. Clinical Medicine Insights: Cardiology, 2016; 10:S37239.

15. MONTEIRO MA, et al. Diabetes and outpatient surgery - protocol in the perioperative period. Revista Portuguesa de Endocrinologia, Diabetes e Metabolismo, 2016, 11:262-267.

16. NAITO R, Takatoshi Kasai. Coronary artery disease in type 2 diabetes mellitus: Recent treatment strategies and future perspectives. World journal of cardiology, 2015; 7(3): 119-124.

17. NAKAMURA M, et al. Three-year follow-up outcomes of SES and PES in a randomized controlled study stratified by the presence of diabetes mellitus: J-DEsSERT trial. International Journal of Cardiology, 2016; 208: 4-12.

18. RAMANATHAN K, et al. Surgical Versus Percutaneous Coronary Revascularization in Patients With Diabetes and Acute Coronary Syndromes. Journal of the American College of Cardiology, 2017; 70(24): 2995-3006.

19. SHROYER A, et al. Comparing Off-Pump and On-Pump Clinical Outcomes and Costs for Diabetic Cardiac Surgery Patients. The Annals of Thoracic Surgery, 2014; 98(1): 38-45.

20. SHROYER A, et al. Off-Pump Versus On-Pump Impact: Diabetic Patient 5-Year Coronary Artery Bypass Clinical Outcomes. The Annals of Thoracic Surgery, 2019; 107(1): 92-98.

21. SINGH A, et al. On-pump versus off-pump coronary artery bypass graft surgery among patients with type 2 diabetes in the Bypass Angioplasty Revascularization Investigation 2 Diabetes trial. Eur J Cardiothorac Surg, 2016; 49(2):40616.

22. WANG TKM, et al. Relationship Between Diabetic Variables and Outcomes After Coronary Artery Bypass Grafting in Diabetic Patients. Heart, Lung and Circulation, 2017; 26(4): 371-375. 